\title{
Systolic inequalities for K3 surfaces via stability conditions
}

\author{
Yu-Wei Fan ${ }^{1}$
}

Received: 24 April 2021 / Accepted: 9 May 2021 / Published online: 19 June 2021

(c) The Author(s) 2021

\section{Abstract}

We introduce the notions of categorical systoles and categorical volumes of Bridgeland stability conditions on triangulated categories. We prove that for any projective K3 surface $X$, there exists a constant $C$ depending only on the rank and discriminant of $N S(X)$, such that

$$
\operatorname{sys}(\sigma)^{2} \leq C \cdot \operatorname{vol}(\sigma)
$$

holds for any stability condition on $\mathcal{D}^{b} \operatorname{Coh}(X)$. This is an algebro-geometric generalization of a classical systolic inequality on two-tori. We also discuss applications of this inequality in symplectic geometry.

Keywords Derived categories · Bridgeland stability conditions · Systolic inequalities · Calabi-Yau manifolds · Mirror symmetry

Mathematics Subject Classification 14F05 (14J33, 18E30, 53D37)

\section{Contents}

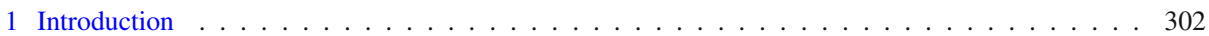

Related work . . . . . . . . . . . . . . . . . . . . . . . . . . . . 304

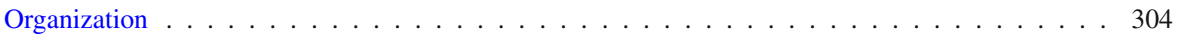

2 Categorical systoles and categorical volumes . . . . . . . . . . . . . . . . . . . . 305

2.1 Bridgeland stability conditions . . . . . . . . . . . . . . . . . . . . . 305

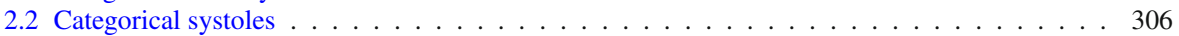

2.3 Categorical volumes . . . . . . . . . . . . . . . . . . . . . . . . 310

3 Systolic inequality for elliptic curves: a sanity check . . . . . . . . . . . . . . . . . . . . . . 312

4 Systolic inequalities for $\mathrm{K} 3$ surfaces . . . . . . . . . . . . . . . . . . . . . . . . . . 314

4.1 Reduction to lattice-theoretic problems . . . . . . . . . . . . . . . . . . . . . . . . . 314

4.2 Systolic inequality for K3 surfaces of Picard rank one . . . . . . . . . . . . . . . . . . . . 317

4.3 Systolic inequality for general K3 surfaces . . . . . . . . . . . . . . . . . . . . . . . . . . . 319

5 Future studies . . . . . . . . . . . . . . . . . . . . . . . . . . 320

Applications in symplectic geometry . . . . . . . . . . . . . . . . . . . . . . 320

Systolic inequality for Calabi-Yau threefolds . . . . . . . . . . . . . . . . . . . . . . . 321

$\bowtie$ Yu-Wei Fan

yuweifanx@gmail.com

1 Department of Mathematics, University of California, Berkeley, Berkeley, CA 94720, USA 
Categorical systole as topological Morse function . . . . . . . . . . . . . . . . . . . . 321

References . . . . . . . . . . . . . . . . . . . . . . 322

\section{Introduction}

Let $(M, g)$ be a Riemannian manifold. Its systole $\operatorname{sys}(M, g)$ is defined to be the least length of a non-contractible loop in $M$. In 1949, Charles Loewner proved that

$$
\text { sys }\left(\mathbb{T}^{2}, g\right)^{2} \leq \frac{2}{\sqrt{3}} \operatorname{vol}\left(\mathbb{T}^{2}, g\right)
$$

holds for any Riemannian metric $g$ on a two-torus $\mathbb{T}^{2}$. There are various generalizations of Loewner's tours systolic inequality. We refer to [16] for a survey on the rich subject of systolic geometry.

The first goal of the present article is to propose a new generalization of Loewner's torus systolic inequality from the perspective of Calabi-Yau geometry. We start with an observation in the case of a two-torus. Suppose that the torus is flat $\mathbb{T}_{\tau}^{2} \cong \mathbb{C} / \mathbb{Z}+\tau \mathbb{Z}$, and is equipped with the standard complex structure $\Omega=d z$ and symplectic structure $\omega=d x \wedge d y$. Then the shortest non-contractible loops must be straight lines, therefore are special Lagrangian submanifolds with respect to the complex and symplectic structures. Under these assumptions, Loewner's torus systolic inequality can be interpreted as:

$$
\inf _{\operatorname{sLag} L \subset \mathbb{T}_{\tau}^{2}}\left|\int_{L} d z\right|^{2} \leq \frac{1}{\sqrt{3}}\left|\int_{\mathbb{T}_{\tau}^{2}} d z \wedge d \bar{z}\right| \text { for all } \tau \in \mathbb{H} .
$$

The key observation is that the quantities in both sides of this inequality can be generalized to any Calabi-Yau manifold.

We propose the following definition of systole of a Calabi-Yau manifold, with respect to its complex and symplectic structures.

Definition 1.1 Let $Y$ be a Calabi-Yau manifold, equipping with a symplectic form $\omega$ and a holomorphic top form $\Omega$. Then its systole is defined to be

$$
\operatorname{sys}(Y, \omega, \Omega):=\inf \left\{\left|\int_{L} \Omega\right|: L \text { is a compact special Lagrangian in }(Y, \omega, \Omega)\right\} .
$$

With this definition, we propose the following question that naturally generalizes inequality (1) to any Calabi-Yau manifold.

Question 1.2 Let $Y$ be a Calabi-Yau manifold and $\omega$ be a symplectic form on $Y$. Does there exist a constant $C=C(Y, \omega)>0$ such that

$$
\operatorname{sys}(Y, \omega, \Omega)^{2} \leq C \cdot\left|\int_{Y} \Omega \wedge \bar{\Omega}\right|
$$

holds for any holomorphic top form $\Omega$ on $Y$ ?

Here we treat the Calabi-Yau manifolds topologically so that the complex structures $\Omega$ can vary. Note that the choice of the symplectic structure is not important in the case of two-tori, since any one-dimensional submanifold is Lagrangian in $\mathbb{T}^{2}$. However, in higher dimensions, the notion of Lagrangian submanifolds certainly depends on the choice of the symplectic structure. Therefore the systolic constant $C$ should depend on the symplectic structure in general, unlike the case of $\mathbb{T}^{2}$. Also, note that the ratio $\left|\int_{L} \Omega\right|^{2} /\left|\int_{Y} \Omega \wedge \bar{\Omega}\right|$ 
has been considered in the context of attractor mechanism in physics [9,20,23], which is of independent interest.

The second goal of the present article is to introduce the definitions of categorical systoles and categorical volumes of Bridgeland stability conditions on triangulated categories.

Definition 1.3 (see Definition 2.4) Let $\mathcal{D}$ be a triangulated category and $\sigma=(\mathcal{Z}, \mathcal{P})$ be a Bridgeland stability condition on $\mathcal{D}$. Its systole is defined to be

$$
\operatorname{sys}(\sigma):=\min \left\{\left|\mathcal{Z}_{\sigma}(E)\right|: E \text { is a } \sigma \text {-semistable object in } \mathcal{D}\right\} .
$$

Note that in the definition of systole of a Bridgeland stability condition, the minimum can always be attained by some $\sigma$-stable object $E$, therefore we can write "min" instead of "inf" (see Remark 2.5).

Definition 1.4 (see Definition 2.11) Let $\left\{E_{i}\right\}$ be a basis of the numerical Grothendieck group $\mathcal{N}(\mathcal{D})$, and $\sigma=(\mathcal{Z}, \mathcal{P})$ be a Bridgeland stability condition on $\mathcal{D}$. Its volume is defined to be

$$
\operatorname{vol}(\sigma):=\left|\sum_{i, j} \chi^{i, j} \mathcal{Z}\left(E_{i}\right) \overline{\mathcal{Z}\left(E_{j}\right)}\right|,
$$

where $\left(\chi^{i, j}\right)=\left(\chi\left(E_{i}, E_{j}\right)\right)^{-1}$ is the inverse matrix of the Euler pairings.

The motivations of these definitions stem from the correspondence between flat surfaces and stability conditions, and the conjectural description of stability conditions on the Fukaya categories of Calabi-Yau manifolds. We refer to Sect. 2 for more details. Note that under these correspondences, $\operatorname{sys}(\sigma)$ is the categorical generalization of $\operatorname{sys}(Y, \omega, \Omega)$ in Definition 1.1 , and $\operatorname{vol}(\sigma)$ is the categorical generalization of the holomorphic volume $\left|\int_{Y} \Omega \wedge \bar{\Omega}\right|$.

As a sanity check of these definitions, we prove the following categorical analogue of Loewner's torus systolic inequality.

Theorem 1.5 (see Theorem 3.1) Let $\mathcal{D}=\mathcal{D}^{b} \operatorname{Coh}(E)$ be the derived category of an elliptic curve E. Then

$$
\operatorname{sys}(\sigma)^{2} \leq \frac{1}{\sqrt{3}} \cdot \operatorname{vol}(\sigma)
$$

holds for any $\sigma \in \operatorname{Stab}(\mathcal{D})$.

We then propose the following algebro-geometric analogue of Question 1.2, which is the higher-dimensional generalization of Theorem 1.5.

Question 1.6 Let X be a Calabi-Yau manifold and $\Omega$ be a complex structure on $X$. Let $\mathcal{D}=\mathcal{D}^{b} \operatorname{Coh}(X, \Omega)$ be its derived category of coherent sheaves. Does there exist a constant $C=C(X, \Omega)>0$ such that

$$
\operatorname{sys}(\sigma)^{2} \leq C \cdot \operatorname{vol}(\sigma)
$$

holds for any $\sigma \in \operatorname{Stab}^{\dagger}(\mathcal{D})$ ? Here $\operatorname{Stab}^{\dagger}(\mathcal{D})$ denotes the distinguished connected component of $\operatorname{Stab}(\mathcal{D})$ that contains geometric stability conditions.

Note that Questions 1.2 and 1.6 are related via the mirror symmetry conjecture, which is a conjectural duality between algebraic geometry and symplectic geometry. The homological mirror symmetry conjecture proposed by Kontsevich [18] states that for any Calabi-Yau manifold with a symplectic structure $(Y, \omega)$, there exists a Calabi-Yau manifold with a 
complex structure $(X, \Omega)$ such that there is an equivalence between the derived Fukaya category of $Y$ and the bounded derived category of coherent sheaves on $X$ :

$$
\mathcal{D}^{b} \operatorname{Fuk}(Y, \omega) \cong \mathcal{D}^{b} \operatorname{Coh}(X, \Omega) .
$$

It is conjectured by Bridgeland [6] and Joyce [15] that a holomorphic top form on $Y$ should give rise to a Bridgeland stability condition $\sigma_{\Omega}$ on $\mathcal{D}^{b} \operatorname{Fuk}(Y, \omega)$ (see Conjecture 2.3). The conjectural stability condition satisfies $\operatorname{sys}\left(\sigma_{\Omega}\right)=\operatorname{sys}(Y, \omega, \Omega)$ and $\operatorname{vol}\left(\sigma_{\Omega}\right)=\left|\int_{Y} \Omega \wedge \bar{\Omega}\right|$. Therefore we can consider Question 1.6 as the mirror counterpart of Question 1.2. We refer to Sect. 5 for more discussions on this.

The third goal, which is the main result of the present article, is to give an affirmative answer to Question 1.6 for any complex projective K3 surface. A priori there is no reason to believe that Question 1.2 and Question 1.6 have affirmative answers in general. The following theorem is the first evidence that the natural categorical generalization of systolic inequality is possible for higher-dimensional Calabi-Yau manifolds.

Theorem 1.7 (see Theorem 4.1) Let X be a complex projective K3 surface. Then

$$
\operatorname{sys}(\sigma)^{2} \leq C \cdot \operatorname{vol}(\sigma)
$$

for any $\sigma \in \operatorname{Stab}^{\dagger}\left(\mathcal{D}^{b} \operatorname{Coh}(X)\right)$, where

$$
C=\frac{((\rho+2) !)^{2}|\operatorname{disc} \mathrm{NS}(X)|}{2^{\rho}}+4 .
$$

Here $\rho$ and disc denote the rank and the discriminant of the Néron-Severi group $\operatorname{NS}(X)$, respectively.

Moreover, when the K3 surface is of Picard rank one, we can use a different method to get a better systolic bound.

Theorem 1.8 (See Theorem 4.2) Let $X$ be a K3 surface of Picard rank one, with $\mathrm{NS}(X)=\mathbb{Z} H$ and $H^{2}=2 n$. Then

$$
\operatorname{sys}(\sigma)^{2} \leq 4(n+1) \cdot \operatorname{vol}(\sigma)
$$

holds for any $\sigma \in \operatorname{Stab}^{\dagger}\left(\mathcal{D}^{b} \operatorname{Coh}(X)\right)$.

Finally, we remark that one can also define a categorical generalization of systole using only the spherical objects (Remark 4.8), which we denoted by $\operatorname{sys}_{\mathrm{sph}}(\sigma)$. However, as we prove in Proposition 4.9, the ratio sys $\operatorname{sph}(\sigma)^{2} / \operatorname{vol}(\sigma)$ is unbounded in general.

\section{Related work}

After the first version of the present article was posted online, Haiden [13] proves a systolic inequality for certain higher-dimensional symplectic torus, and Pacini [25] proposes a higherdimensional generalization of extremal lengths and complex systolic inequalities.

\section{Organization}

In Sect. 2, we recall the definition of Bridgeland stability conditions and introduce the notions of categorical systole and categorical volume. In Sect. 3, we give an affirmative answer to 
Question 1.6 for elliptic curves by proving Theorem 1.5. In Sect. 4, we give an affirmative answer to Question 1.6 for any K3 surface by proving Theorem 1.7. In Sect. 5, we discuss some directions for future studies.

\section{Categorical systoles and categorical volumes}

\subsection{Bridgeland stability conditions}

In the seminal work [4], Bridgeland introduced the notion of stability conditions on triangulated categories. We recall the definition and some basic properties of Bridgeland stability conditions.

Throughout the article, a triangulated category $\mathcal{D}$ is essentially small, linear over $\mathbb{C}$, and is of finite type. The last condition means that for any pair of objects $E, F \in \mathcal{D}$, the $\mathbb{C}$-vector space $\oplus_{i \in \mathbb{Z}} \operatorname{Hom}_{\mathcal{D}}(E, F[i])$ is of finite-dimensional. The Euler form $\chi$ on the Grothendieck group $K(\mathcal{D})$ is given by the alternating sum

$$
\chi(E, F):=\sum_{i}(-1)^{i} \operatorname{dim}_{\mathbb{C}} \operatorname{Hom}_{\mathcal{D}}(E, F[i]) .
$$

The numerical Grothendieck group $\mathcal{N}(\mathcal{D}):=K(\mathcal{D}) / K(\mathcal{D})^{\perp_{\chi}}$ is defined to be the quotient of $K(\mathcal{D})$ by the null space of the Euler pairing $\chi$. We assume that $\mathcal{D}$ is numerically finite, that is, $\mathcal{N}(\mathcal{D})$ is of finite rank. One large class of examples of such triangulated categories is provided by the bounded derived category of coherent sheaves $\mathcal{D}^{b} \operatorname{Coh}(X)$ of a smooth projective variety $X$.

Definition 2.1 [4] A (full numerical) stability condition $\sigma=(\mathcal{Z}, \mathcal{P})$ on a triangulated category $\mathcal{D}$ consists of:

- a group homomorphism $\mathcal{Z}: \mathcal{N}(\mathcal{D}) \rightarrow \mathbb{C}$, and

- a collection of full additive subcategories $\mathcal{P}=\{\mathcal{P}(\phi)\}_{\phi \in \mathbb{R}}$ of $\mathcal{D}$,

such that:

(a) If $0 \neq E \in \mathcal{P}(\phi)$, then $\mathcal{Z}(E) \in \mathbb{R}_{>0} \cdot e^{i \pi \phi}$.

(b) $\mathcal{P}(\phi+1)=\mathcal{P}(\phi)[1]$.

(c) If $\phi_{1}>\phi_{2}$ and $A_{i} \in \mathcal{P}\left(\phi_{i}\right)$, then $\operatorname{Hom}\left(A_{1}, A_{2}\right)=0$.

(d) For every $0 \neq E \in \mathcal{D}$, there exists a (unique) collection of distinguished triangles

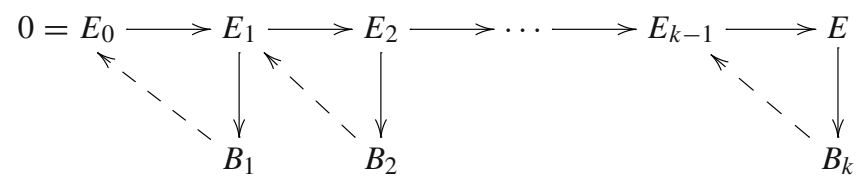

such that $B_{i} \in \mathcal{P}\left(\phi_{i}\right)$ and $\phi_{1}>\phi_{2}>\cdots>\phi_{k}$. Denote $\phi_{\sigma}^{+}(E):=\phi_{1}$ and $\phi_{\sigma}^{-}(E):=\phi_{k}$. The mass of $E$ is defined to be $m_{\sigma}(E):=\sum_{i}\left|\mathcal{Z}\left(B_{i}\right)\right|$.

(e) (Support property [19]) There exists a constant $C>0$ and a norm $\|\cdot\|$ on $\mathcal{N}(\mathcal{D}) \otimes_{\mathbb{Z}} \mathbb{R}$ such that

$$
\|E\| \leq C|\mathcal{Z}(E)|
$$

for any semistable object $E$. 
The group homomorphism $\mathcal{Z}$ is called the central charge, and the nonzero objects in $\mathcal{P}(\phi)$ are called the semistable objects of phase $\phi$. The additive subcategories $\mathcal{P}(\phi)$ actually are abelian, and the simple objects of $\mathcal{P}(\phi)$ are said to be stable.

The space of (full numerical) Bridgeland stability conditions on $\mathcal{D}$ is denoted by $\operatorname{Stab}(\mathcal{D})$. There is a nice topology on $\operatorname{Stab}(\mathcal{D})$ introduced by Bridgeland, which is induced by the generalized distance:

$d\left(\sigma_{1}, \sigma_{2}\right)=\sup _{0 \neq E \in \mathcal{D}}\left\{\left|\phi_{\sigma_{2}}^{-}(E)-\phi_{\sigma_{1}}^{-}(E)\right|,\left|\phi_{\sigma_{2}}^{+}(E)-\phi_{\sigma_{1}}^{+}(E)\right|,\left|\log \frac{m_{\sigma_{2}}(E)}{m_{\sigma_{1}}(E)}\right|\right\} \in[0, \infty]$.

The forgetful map

$$
\operatorname{Stab}(\mathcal{D}) \longrightarrow \operatorname{Hom}(\mathcal{N}(\mathcal{D}), \mathbb{C}), \quad \sigma=(\mathcal{Z}, \mathcal{P}) \mapsto \mathcal{Z}
$$

is a local homeomorphism $[4,19]$. Hence $\operatorname{Stab}(\mathcal{D})$ is a complex manifold.

There are two natural group actions on the space of Bridgeland stability conditions $\operatorname{Stab}(\mathcal{D})$ which commute with each other [4, Lemma 8.2]. Firstly, the group of autoequivalences $\operatorname{Aut}(\mathcal{D})$ acts on $\operatorname{Stab}(\mathcal{D})$ as isometries with respect to the generalized metric: Let $\Phi \in \operatorname{Aut}(\mathcal{D})$ be an autoequivalence, define

$$
\sigma=(\mathcal{Z}, \mathcal{P}) \mapsto \Phi \cdot \sigma:=\left(\mathcal{Z} \circ[\Phi]^{-1}, \mathcal{P}^{\prime}\right),
$$

where $[\Phi]$ is the induced automorphism on $\mathcal{N}(\mathcal{D})$, and $\mathcal{P}^{\prime}(\phi):=\Phi(\mathcal{P}(\phi))$.

Secondly, the universal cover $\widehat{\mathrm{GL}^{+}(2, \mathbb{R})}$ also admits a natural group action on $\operatorname{Stab}(\mathcal{D})$. Recall that $\widetilde{\mathrm{GL}^{+}(2, \mathbb{R})}$ is isomorphic to the group of pairs $(T, f)$, where $T \in \mathrm{GL}^{+}(2, \mathbb{R})$ and $f: \mathbb{R} \rightarrow \mathbb{R}$ is an increasing map with $f(\phi+1)=f(\phi)+1$, such that their induced maps on $\left(\mathbb{R}^{2} \backslash\{0\}\right) / \mathbb{R}_{>0} \cong \mathbb{R} / 2 \mathbb{Z} \cong S^{1}$ coincide. Let $g=(T, f) \in \mathrm{GL}^{+}(2, \mathbb{R})$, define

$$
\sigma=(\mathcal{Z}, \mathcal{P}) \mapsto \sigma \cdot g:=\left(T^{-1} \circ \mathcal{Z}, \mathcal{P}^{\prime \prime}\right),
$$

where $\mathcal{P}^{\prime \prime}(\phi):=\mathcal{P}(f(\phi))$. Note that the subgroup $\left.\mathbb{C} \subset \widetilde{\mathrm{GL}^{+}(2,}, \mathbb{R}\right)$ acts freely and transitively on $\operatorname{Stab}(\mathcal{D})$. Let $z \in \mathbb{C}$, then its action on $\operatorname{Stab}(\mathcal{D})$ is

$$
\sigma=(\mathcal{Z}, \mathcal{P}) \mapsto \sigma \cdot z:=\left(\exp (-i \pi z) \mathcal{Z}, \mathcal{P}^{\prime \prime \prime}\right),
$$

where $\mathcal{P}^{\prime \prime \prime}(\phi):=\mathcal{P}(\phi+\operatorname{Re}(z))$.

\subsection{Categorical systoles}

We first recall some results and conjectures that motivate our definition of categorical systoles.

In a striking series of work by Gaiotto-Moore-Neitzke [12], Bridgeland-Smith [7] and Haiden-Katzarkov-Kontsevich [14], the connections between stability conditions and Teichmüller theory have been established. One of the main results in this direction is the following theorem.

Theorem 2.2 ([14], Theorem 5.2 and 5.3) Let $S$ be a marked surface of finite type, $\mathcal{M}(S)$ be the space of marked flat structures on $S$, and $\operatorname{Fuk}(S)$ be the Fukaya category of $S$. Then there is a natural map

$$
\mathcal{M}(S) \rightarrow \operatorname{Stab}(\operatorname{Fuk}(S))
$$

which is bianalytic onto its image, which is a union of connected components. Under this bianalytic map, 
- saddle connections on flat surfaces are corresponded to semistable objects,

- their lengths are corresponded to the absolute values of central charges.

Recall that the systole of a flat surface is defined to be the length of its shortest saddle connection. Based on the correspondence established in Theorem 2.2, it is natural to define the systole of a Bridgeland stability condition to be the smallest absolute value of central charge of semistable objects.

Another important source of motivation for defining categorical systole is a conjectural description of stability conditions on the Fukaya categories of Calabi-Yau manifolds, proposed by Bridgeland and Joyce.

Conjecture 2.3 ([6], Section 2.4 and [15], Conjecture 3.2) Let $Y$ be a Calabi-Yau manifold equipping with a symplectic form $\omega$, and $\mathcal{D}^{\pi} \operatorname{Fuk}(Y, \omega)$ be the derived Fukaya category of $Y$. For any holomorphic top form $\Omega$ on $Y$, there exists a natural Bridgeland stability condition $\sigma_{\Omega}$ on $\mathcal{D}^{\pi} \mathrm{Fuk}(Y, \omega)$, such that the central charges are given by the period integrals along Lagrangians

$$
\mathcal{Z}_{\Omega}(L)=\int_{L} \Omega
$$

and the $\sigma_{\Omega}$-semistable objects are given by compact special Lagrangian submanifolds with respect to $\Omega$ and $\omega$.

If we assume this conjecture to be true, then the definition of systole of $(Y, \omega, \Omega)$ in Definition 1.1 can be written as

$$
\begin{aligned}
\operatorname{sys}(Y, \omega, \Omega) & :=\inf \left\{\left|\int_{L} \Omega\right|: L \text { is a compact special Lagrangian in }(Y, \omega, \Omega)\right\} \\
& =\inf \left\{|\mathcal{Z}(L)|: L \text { is a } \sigma_{\Omega} \text {-semistable object } \operatorname{in} \mathcal{D}^{\pi} \operatorname{Fuk}(Y, \omega)\right\}
\end{aligned}
$$

Motivating from the above discussions, we propose the following definition of systole of a Bridgeland stability condition.

Definition 2.4 Let $\sigma$ be a Bridgeland stability condition on a triangulated category $\mathcal{D}$. Its systole is defined to be

$$
\operatorname{sys}(\sigma):=\min \left\{\left|\mathcal{Z}_{\sigma}(E)\right|: E \text { is a } \sigma \text {-semistable object in } \mathcal{D}\right\} .
$$

Remark 2.5 Note that we can write "min" instead of "inf" in the definition of categorical systole for the following reason. For any $R>0$, consider the following two subsets of $\mathcal{N}(\mathcal{D})$ :

$S_{R}^{(1)}:=\left\{v \in \mathcal{N}(\mathcal{D})\right.$ : there exists $\sigma$-semistable $E$ such that $[E]=v$ and $\left.\left|\mathcal{Z}_{\sigma}(v)\right|<R\right\}$,

$S_{R}^{(2)}:=\{v \in \mathcal{N}(\mathcal{D})$ : there exists $\sigma$-semistable $E$ such that $[E]=v$ and $\|v\|<C R\}$,

where $C>0$ and $\|\cdot\|$ are the constant and the norm on $\mathcal{N}(\mathcal{D}) \otimes_{\mathbb{Z}} \mathbb{R}$ appeared in the support property of stability conditions, see Definition 2.12.1. By the support property, we have $S_{R}^{(1)} \subset S_{R}^{(2)}$. The set $S_{R}^{(2)}$ is finite since it is a subset of $\{v \in \mathcal{N}(\mathcal{D}):\|v\|<C R\}$ which is finite. Therefore $S_{R}^{(1)}$ is a finite set, which implies that the minimum of

$$
\left\{\left|\mathcal{Z}_{\sigma}(E)\right|: E \text { is a } \sigma \text {-semistable object in } \mathcal{D}\right\}
$$

in the definition of systole must be attained by some $\sigma$-semistable object $E$. In fact, the systole must be attained by some $\sigma$-stable object: if $E$ is a $\sigma$-semistable object and not a 
$\sigma$-stable object, then it is clear that the absolute value of the central charge of any non-trivial $\sigma$-stable factor of $E$ is strictly less than $\left|Z_{\sigma}(E)\right|$.

Remark 2.6 In the following table, we summarize the correspondences between the systoles of flat surfaces, Calabi-Yau manifolds, and stability conditions discussed in Theorem 2.2 and Conjecture 2.3.

\begin{tabular}{lll}
\hline Surface $S$ & Calabi-Yau $(Y, \omega)$ & Triangulated category \\
\hline abelian differentials & holomorphic top forms & stability conditions \\
saddle connections & special Lagrangians & semistable objects \\
lengths & period integrals & central charges \\
sys $(S)$ & sys $(Y, \omega, \Omega)$ & sys $(\sigma)$ \\
& $\left|\int_{Y} \Omega \wedge \bar{\Omega}\right|$ & $\operatorname{vol}(\sigma)$ \\
\hline
\end{tabular}

The definition of categorical volume $\operatorname{vol}(\sigma)$, which is also inspired by Conjecture 2.3, will be discussed in the next subsection.

Now we study how the categorical systole changes under the natural group actions on $\operatorname{Stab}(\mathcal{D})$.

Lemma 2.7 Let $\sigma$ be a Bridgeland stability condition on $\mathcal{D}$. Then

(a)

$$
\begin{aligned}
\operatorname{sys}(\sigma) & =\min \left\{\left|\mathcal{Z}_{\sigma}(E)\right|: E \text { is a } \sigma \text {-stable object in } \mathcal{D}\right\} \\
& =\min \left\{m_{\sigma}(E): 0 \neq E \in \mathcal{D}\right\}
\end{aligned}
$$

Recall that $m_{\sigma}(E)$ is the mass of $E$ with respect to $\sigma$ (Definition 2.1).

(b) For any autoequivalence $\Phi \in \operatorname{Aut}(\mathcal{D})$, $\operatorname{sys}(\Phi \cdot \sigma)=\operatorname{sys}(\sigma)$.

(c) For any complex number $z=x+i y \in \mathbb{C}$, $\operatorname{sys}(\sigma \cdot z)=\exp (y \pi) \cdot \operatorname{sys}(\sigma)$.

(d) For any $g=(T, f) \in \mathrm{GL}^{+}(2, \mathbb{R})$, write $T^{-1}=\left[\begin{array}{ll}a & b \\ c & d\end{array}\right] \in \mathrm{GL}^{+}(2, \mathbb{R})$, then

$$
\operatorname{sys}(\sigma \cdot g) \leq\left|t_{1}\right|\left(1+\left|t_{2}\right|\right) \cdot \operatorname{sys}(\sigma),
$$

where $t_{1}=\frac{(a+d)+i(c-b)}{2} \neq 0$ and $t_{2}=\frac{(a-d)+i(b+c)}{(a+d)+i(c-b)}$. Note that $\left|t_{2}\right|<1$.

Proof The first three statements follow easily from the definition. To prove the last statement, we use a similar idea in the proof of [13, Theorem 4.3]. One can verify by direct computation that

$$
\mathcal{Z}_{\sigma \cdot g}(E)=t_{1}\left(\mathcal{Z}_{\sigma}(E)+t_{2} \overline{\mathcal{Z}_{\sigma}(E)}\right)
$$

holds for any $\sigma$ and $E$. By Remark 2.5, there exists a $\sigma$-stable object $E$ such that $\operatorname{sys}(\sigma)=$ $\left|\mathcal{Z}_{\sigma}(E)\right|$. Since the actions by $\mathrm{GL}^{+}(2, \mathbb{R})$ do not change the set of stable objects, $E$ is also $\sigma \cdot g$-stable. Therefore,

$$
\begin{aligned}
\left|t_{1}\right|\left(1+\left|t_{2}\right|\right) \cdot \operatorname{sys}(\sigma) & =\left|t_{1}\right|\left(1+\left|t_{2}\right|\right) \cdot\left|\mathcal{Z}_{\sigma}(E)\right| \\
& \geq\left|\mathcal{Z}_{\sigma \cdot g}(E)\right| \geq \operatorname{sys}(\sigma \cdot g)
\end{aligned}
$$


Proposition 2.8 The function

$$
\text { sys: } \operatorname{Stab}(\mathcal{D}) \longrightarrow \mathbb{R}_{>0}, \quad \sigma \mapsto \operatorname{sys}(\sigma)
$$

is continuous on $\operatorname{Stab}(\mathcal{D})$.

Proof Let $\sigma_{1}, \sigma_{2} \in \operatorname{Stab}(\mathcal{D})$ be two Bridgeland stability conditions with $d\left(\sigma_{1}, \sigma_{2}\right)<\epsilon$. By Remark 2.5, sys $\left(\sigma_{1}\right)=m_{\sigma_{1}}(E)$ for some object $E \in \mathcal{D}$. Thus

$$
\log \operatorname{sys}\left(\sigma_{1}\right)=\log m_{\sigma_{1}}(E)>\log m_{\sigma_{2}}(E)-\epsilon \geq \log \operatorname{sys}\left(\sigma_{2}\right)-\epsilon .
$$

Similarly, we have $\log \operatorname{sys}\left(\sigma_{2}\right)>\log \operatorname{sys}\left(\sigma_{1}\right)-\epsilon$. Hence $\log \operatorname{sys}(\sigma)$ is a continuous function on $\operatorname{Stab}(\mathcal{D})$, and so is $\operatorname{sys}(\sigma)$.

Let us consider some basic examples of categorical systoles of algebraic stability conditions. In Sects. 3 and 4, we will be dealing with non-algebraic stability conditions on derived categories of coherent sheaves.

Example 2.9 (Derived category of representations of $A_{2}$-quiver) Let $Q$ be the $A_{2}$-quiver $(\cdot \rightarrow$ -) and $\mathcal{D}=\mathcal{D}^{b}(\operatorname{Rep}(Q))$ be the bounded derived category of the category of representations of $Q$. Let $E_{1}$ and $E_{2}$ be the simple objects in $\operatorname{Rep}(Q)$ with dimension vectors $\underline{\operatorname{dim}}\left(E_{1}\right)=(1,0)$ and $\underline{\operatorname{dim}}\left(E_{2}\right)=(0,1)$. There is one more indecomposable object $E_{3}$ that fits into the exact sequence

$$
0 \rightarrow E_{2} \rightarrow E_{3} \rightarrow E_{1} \rightarrow 0
$$

Let $\sigma=(\mathcal{Z}, \mathcal{P})$ be a stability condition on $\mathcal{D}$ with $z_{1}:=\mathcal{Z}\left(E_{1}\right)$ and $z_{2}:=\mathcal{Z}\left(E_{2}\right)$. Suppose that $\mathcal{P}(0,1]=\operatorname{Rep}(Q)$. Then

- When $\arg \left(z_{1}\right)<\arg \left(z_{2}\right)$, the only $\sigma$-stable objects are $E_{1}$ and $E_{2}$ up to shiftings. Thus $\operatorname{sys}(\sigma)=\min \left\{\left|z_{1}\right|,\left|z_{2}\right|\right\}$.

- When $\arg \left(z_{1}\right)>\arg \left(z_{2}\right)$, the only $\sigma$-stable objects are $E_{1}, E_{2}$ and $E_{3}$ up to shiftings. Thus $\operatorname{sys}(\sigma)=\min \left\{\left|z_{1}\right|,\left|z_{2}\right|,\left|z_{1}+z_{2}\right|\right\}$.

Therefore, in order to compute the categorical systoles of stability conditions in different chambers, one needs to compute the central charges of different sets of dimensional vectors. Note that this is not the case for the derived categories of elliptic curves and K3 surfaces, where the categorical systole of any stability condition is the minimum of absolute values of central charges of the same set of classes in $\mathcal{N}(\mathcal{D})$ (see Sect. 3 and Proposition 4.4).

Example 2.10 (3-Calabi-Yau categories arising from marked Riemann surfaces) There is a 3-Calabi-Yau category $\mathcal{D}_{S, M}$ (up to equivalence) associated to each marked Riemann surface $(S, M)$, see for instance [7, Section 9]. The main theorem in [7] states that

$$
\operatorname{Stab}\left(\mathcal{D}_{S, M}\right) / \operatorname{Aut}\left(\mathcal{D}_{S, M}\right) \cong \operatorname{Quad}(S, M),
$$

where $\operatorname{Quad}(S, M)$ is the moduli space of certain meromorphic quadratic differentials on $S$ with simple zeros. By Lemma $2.7(2)$, the function sys on $\operatorname{Stab}(\mathcal{D})$ given by categorical systole descends to a function on the quotient $\operatorname{Stab}(\mathcal{D}) / \operatorname{Aut}(\mathcal{D})$. Under the equivalence (3), the categorical systole of a stability condition on $\mathcal{D}_{S, M}$ is given by the minimum among the period integrals $\int \sqrt{\phi}$ along saddle connections of the corresponding quadratic differential. 


\subsection{Categorical volumes}

We recall the notion of categorical volumes of Bridgeland stability conditions introduced in [10]. It is the categorical analogue of the holomorphic volume $\left|\int_{Y} \Omega \wedge \bar{\Omega}\right|$ of a compact Calabi-Yau manifold $Y$ with holomorphic top form $\Omega$.

Let $Y$ be a compact Calabi-Yau manifold of dimension $n$, and $\left\{A_{i}\right\}$ be a basis of the torsion-free part of $H_{n}(X, \mathbb{Z})$. Then one can rewrite the holomorphic volume of $Y$ as

$$
\left|\int_{Y} \Omega \wedge \bar{\Omega}\right|=\left|\sum_{i, j} \gamma^{i, j} \int_{A_{i}} \Omega \int_{A_{j}} \bar{\Omega}\right|,
$$

where $\left(\gamma^{i, j}\right)=\left(A_{i} \cdot A_{j}\right)^{-1}$ is the inverse matrix of the intersection pairings.

Recall that in Conjecture 2.3, the period integral $\int \Omega$ should give the central charge of a Bridgeland stability condition on the derived Fukaya category $\mathcal{D}^{\pi} \operatorname{Fuk}(Y)$ that is associated to the holomorphic top form $\Omega$. This motivates the following definition.

Definition 2.11 [10] Let $\left\{E_{i}\right\}$ be a basis of the numerical Grothendieck group $\mathcal{N}(\mathcal{D})$ and let $\sigma=(\mathcal{Z}, \mathcal{P})$ be a Bridgeland stability condition on $\mathcal{D}$. Its volume is defined to be

$$
\operatorname{vol}(\sigma):=\left|\sum_{i, j} \chi^{i, j} \mathcal{Z}\left(E_{i}\right) \overline{\mathcal{Z}\left(E_{j}\right)}\right|
$$

where $\left(\chi^{i, j}\right)=\left(\chi\left(E_{i}, E_{j}\right)\right)^{-1}$ is the inverse matrix of the Euler pairings.

One can easily check that the above definition is independent of the choice of the basis $\left\{E_{i}\right\}$ : Suppose $\left\{F_{j}\right\}$ is another basis of $\mathcal{N}(\mathcal{D})$, where $A$ is the unimodular matrix that relates the bases $\left\{E_{i}\right\}$ and $\left\{F_{j}\right\}$, i.e. $F_{j}=\sum_{k} A_{j k} E_{k}$. Denote the Gram matrices $\left(\chi\left(E_{i}, E_{j}\right)\right)$ and $\left(\chi\left(F_{i}, F_{j}\right)\right)$ by $\chi_{E}$ and $\chi_{F}$, respectively. Then we have $\chi_{F}=A \chi_{E} A^{T}$, and therefore

$$
\begin{aligned}
\sum_{i, j}\left(\chi_{F}\right)_{i j}^{-1} \mathcal{Z}\left(F_{i}\right) \overline{\mathcal{Z}\left(F_{j}\right)} & =\sum_{i, j}\left(A \chi_{E} A^{T}\right)_{i j}^{-1} \mathcal{Z}\left(F_{i}\right) \overline{\mathcal{Z}\left(F_{j}\right)} \\
& =\sum_{i, j, k, \ell}\left(A^{T}\right)_{i k}^{-1}\left(\chi_{E}\right)_{k \ell}^{-1} A_{\ell j}^{-1} \mathcal{Z}\left(F_{i}\right) \overline{\mathcal{Z}\left(F_{j}\right)} \\
& =\sum_{i, j, k, \ell, m, n} A_{k i}^{-1}\left(\chi_{E}\right)_{k \ell}^{-1} A_{\ell j}^{-1} A_{i m} A_{j n} \mathcal{Z}\left(E_{m}\right) \overline{\mathcal{Z}\left(E_{n}\right)} \\
& =\sum_{k, \ell, m, n} \delta_{k m} \delta_{\ell n}\left(\chi_{E}\right)_{k \ell}^{-1} \mathcal{Z}\left(E_{m}\right) \overline{\mathcal{Z}\left(E_{n}\right)} \\
& =\sum_{m, n}\left(\chi_{E}\right)_{m n}^{-1} \mathcal{Z}\left(E_{m}\right) \overline{\mathcal{Z}\left(E_{n}\right)}
\end{aligned}
$$

It is important to note that unlike the categorical systoles, the categorical volume of a stability condition $\sigma=(\mathcal{Z}, \mathcal{P})$ depends only on its central charge. The proof of the following lemma is straightforward.

\section{Lemma 2.12 Let $\sigma$ be a Bridgeland stability condition on $\mathcal{D}$. Then}

(a) For any autoequivalence $\Phi \in \operatorname{Aut}(\mathcal{D}), \operatorname{vol}(\Phi \cdot \sigma)=\operatorname{vol}(\sigma)$.

(b) For any complex number $z=x+i y \in \mathbb{C}, \operatorname{vol}(\sigma \cdot z)=\exp (2 y \pi) \cdot \operatorname{vol}(\sigma)$. 
Now we recall some computations of categorical volumes in [10] which will be used in the later sections.

Example 2.13 (Elliptic curves) Let $\mathcal{D}=\mathcal{D}^{b} \operatorname{Coh}(E)$ be the derived category of coherent sheaves on an elliptic curve $E$. Let $\sigma=(\mathcal{Z}, \mathcal{P})$ be a stability condition on $\mathcal{D}$ with central charge

$$
\mathcal{Z}_{\sigma}(F)=-\operatorname{deg}(F)+(\beta+i \omega) \cdot \operatorname{rk}(F),
$$

where $\beta \in \mathbb{R}$ and $\omega>0$. Choose $\left\{\mathcal{O}_{x}, \mathcal{O}_{E}\right\}$ as a basis of the numerical Grothendieck group $\mathcal{N}(\mathcal{D})$, where $\mathcal{O}_{x}$ is a skyscraper sheaf and $\mathcal{O}_{E}$ is the structure sheaf. Then the categorical volume of $\sigma$ is

$$
\begin{aligned}
\operatorname{vol}(\sigma) & =\left|\mathcal{Z}_{\sigma}\left(\mathcal{O}_{x}\right) \overline{\mathcal{Z}_{\sigma}\left(\mathcal{O}_{E}\right)}-\mathcal{Z}_{\sigma}\left(\mathcal{O}_{E}\right) \overline{\mathcal{Z}_{\sigma}\left(\mathcal{O}_{x}\right)}\right| \\
& =2 \omega>0
\end{aligned}
$$

Remark 2.14 Let $C$ be a curve of genus $g \geq 1$. Then $\operatorname{Stab}\left(\mathcal{D}^{b} \operatorname{Coh}(C)\right)=\mathbb{C} \times \mathbb{H}$, and the central charge of any stability condition is of the form

$$
\mathcal{Z}_{\sigma}(F)=-\operatorname{deg}(F)+(\beta+i \omega) \cdot \operatorname{rk}(F) \quad(\beta \in \mathbb{R} \text { and } \omega>0)
$$

up to the free $\mathbb{C}$-action (see $[4,22]$ ). However, the categorical volume $\operatorname{vol}(\sigma)$ is not given by $2 \omega$ unless $g=1$, due to the fact that the Euler pairing on $\mathcal{N}(\mathcal{D})$ is not (anti)symmetric if $C$ is not Calabi-Yau. In fact, choose $\left\{E_{1}=\mathcal{O}, E_{2}=\mathcal{O}(1)\right\}$ as a basis of $\mathcal{N}(\mathcal{D})$, then the Gram matrix of Euler pairings is $A:=\left[\begin{array}{cc}1-g & 2-g \\ -g & 1-g\end{array}\right]$ by Riemann-Roch. Therefore we have

$$
\begin{aligned}
\operatorname{vol}(\sigma)= & \left|\sum_{1 \leq i, j \leq 2}\left(A^{-1}\right)_{i j} \mathcal{Z}\left(E_{i}\right) \overline{\mathcal{Z}\left(E_{j}\right)}\right| \\
= & |(1-g)| \beta+\left.i \omega\right|^{2}+(g-2)(\beta+i \omega) \overline{\beta+i \omega-1} \\
& \quad+g(\beta+i \omega-1) \overline{\beta+i \omega}+(1-g)|\beta+i \omega-1|^{2} \mid \\
= & |2 i \omega+(1-g)| \\
= & \sqrt{(g-1)^{2}+(2 \omega)^{2}}>0 .
\end{aligned}
$$

On the other hand, there exist stability conditions on $\mathcal{D}^{b} \operatorname{Coh}\left(\mathbb{P}^{1}\right)$ that are not of this form (non-geometric). It is possible for such stability conditions to have zero categorical volume. For instance, by [24, Proposition 3.3], there exist stability conditions $\sigma \in \operatorname{Stab}\left(\mathcal{D}^{b} \operatorname{Coh}\left(\mathbb{P}^{1}\right)\right)$ such that $\mathcal{Z}_{\sigma}(\mathcal{O})=\mathcal{Z}_{\sigma}(\mathcal{O}(1))=z \in \mathbb{C} \backslash\{0\}$. Then by the same computation as above, we have

$$
\operatorname{vol}(\sigma)=\left.|| z\right|^{2}-2|z|^{2}+|z|^{2} \mid=0
$$

Example 2.15 (K3 surfaces) Let $\mathcal{D}=\mathcal{D}^{b} \mathrm{Coh}(X)$ be the derived category of coherent sheaves on a K3 surface $X$. Let $\sigma=(\mathcal{Z}, \mathcal{P})$ be a stability condition on $\mathcal{D}$ with central charge

$$
\mathcal{Z}_{\sigma}(v)=(\exp (\beta+i \omega), v)
$$

where $(\cdot, \cdot)$ is the Mukai pairing on the numerical Grothendieck group $\mathcal{N}(\mathcal{D})$, and $\beta, \omega \in$ $\mathrm{NS}(X) \otimes \mathbb{R}$ with $\omega^{2}>0$ (c.f. Section 4). Let $\left\{v_{i}\right\}$ be a basis of $\mathcal{N}(\mathcal{D})$. Then the categorical 
volume of $\sigma$ is

$$
\begin{aligned}
\operatorname{vol}(\sigma) & =\left|\sum_{i, j} \chi^{i, j} \mathcal{Z}_{\sigma}\left(v_{i}\right) \overline{\mathcal{Z}_{\sigma}\left(v_{j}\right)}\right| \\
& =\left|\sum_{i, j} \chi^{i, j} \cdot\left(\exp (\beta+i \omega), v_{i}\right) \cdot\left(\exp (\beta-i \omega), v_{j}\right)\right| \\
& =\left|\sum_{i, j}\left(\exp (\beta+i \omega), v_{i}\right) \cdot \chi^{i, j} \cdot\left(v_{j}, \exp (\beta-i \omega)\right)\right| \quad \text { (Serre duality) } \\
& =|(\exp (\beta+i \omega), \exp (\beta-i \omega))| \quad(\text { compatibility btw Euler/Mukai pairings) } \\
& =2 \omega^{2}>0 .
\end{aligned}
$$

Note that the same computation shows that $\sum_{i, j} \chi^{i, j} \mathcal{Z}_{\sigma}\left(v_{i}\right) \mathcal{Z}_{\sigma}\left(v_{j}\right)=0$.

For any $g=(T, f) \in \mathrm{GL}^{+}(2, \mathbb{R})$, one can compute the categorical volume of the stability condition $\sigma \cdot g$ following the same idea in the proof of Lemma 2.7 (4). Write $T^{-1}=\left[\begin{array}{ll}a & b \\ c & d\end{array}\right] \in \mathrm{GL}^{+}(2, \mathbb{R})$. Define $t_{1}=\frac{(a+d)+i(c-b)}{2} \neq 0$ and $t_{2}=\frac{(a-d)+i(b+c)}{(a+d)+i(c-b)}$. By Eq. (2), we have

$$
\begin{aligned}
\operatorname{vol}(\sigma \cdot g) & =\left|\sum_{i, j} \chi^{i, j} \mathcal{Z}_{\sigma \cdot g}\left(v_{i}\right) \overline{\mathcal{Z}_{\sigma \cdot g}\left(v_{j}\right)}\right| \\
& =\left|t_{1}\right|^{2}\left|\sum_{i, j} \chi^{i, j}\left(\mathcal{Z}_{\sigma}\left(v_{i}\right)+t_{2} \overline{\mathcal{Z}_{\sigma}\left(v_{i}\right)}\right)\left(\overline{\mathcal{Z}_{\sigma}\left(v_{j}\right)}+\overline{t_{2}} \mathcal{Z}_{\sigma}\left(v_{j}\right)\right)\right| \\
& =\left|t_{1}\right|^{2}\left(1+\left|t_{2}\right|^{2}\right) \cdot \operatorname{vol}(\sigma) .
\end{aligned}
$$

We use $\chi^{i, j}=\chi^{j, i}$ in the last step, which follows from the fact that $\mathcal{D}$ is a 2-Calabi-Yau category therefore its Euler pairing is symmetric.

We should note that although the categorical volume can be defined for stability conditions on any triangulated category $\mathcal{D}$, its geometric meaning is not clear unless $\mathcal{D}$ comes from compact Calabi-Yau geometry. Below is an example of a Calabi-Yau triangulated category for which the categorical volume vanishes for some stability conditions (on the wall of marginal stability conditions).

Example 2.16 (3-Calabi-Yau category of the $A_{2}$-quiver) Let $\mathcal{D}$ be the 3-Calabi-Yau category constructed from the Ginzburg 3-Calabi-Yau dg-algebra associated to the $A_{2}$-quiver [11,17]. The numerical Grothendieck group $\mathcal{N}(\mathcal{D})$ is generated by two spherical objects $S_{1}, S_{2}$.

Let $\sigma=(\mathcal{Z}, \mathcal{P})$ be a stability condition on $\mathcal{D}$ with $z_{1}=\mathcal{Z}\left(S_{1}\right)$ and $z_{2}=\mathcal{Z}\left(S_{2}\right)$. Then its categorical volume is

$$
\operatorname{vol}(\sigma)=\left|z_{1} \overline{z_{2}}-z_{2} \overline{z_{1}}\right|=2\left|\operatorname{Im}\left(z_{1} \overline{z_{2}}\right)\right|,
$$

which vanishes if $z_{1} \overline{z_{2}} \in \mathbb{R}$. This can happen if $z_{1}$ and $z_{2}$ are of the same phase, i.e., the stability condition $\sigma$ sits on a wall in $\operatorname{Stab}(\mathcal{D})$.

\section{Systolic inequality for elliptic curves: a sanity check}

In this section, we give an affirmative answer to Question 1.6 in the case of elliptic curves. This provides a sanity check for our definitions of categorical systole and categorical volume. 
Theorem 3.1 Let $\mathcal{D}=\mathcal{D}^{b} \operatorname{Coh}(E)$ be the derived category of an elliptic curve E. Then

$$
\operatorname{sys}(\sigma)^{2} \leq \frac{1}{\sqrt{3}} \cdot \operatorname{vol}(\sigma)
$$

holds for any $\sigma \in \operatorname{Stab}(\mathcal{D})$.

One can consider this inequality as the mirror of Loewner's torus systolic inequality in the introduction. We refer to Sect. 5 for more discussions related to mirror symmetry.

Proof By [4, Theorem 9.1], the $\left.\widetilde{\mathrm{GL}^{+}(2 ;}, \mathbb{R}\right)$-action on the space of Bridgeland stability conditions $\operatorname{Stab}(\mathcal{D})$ of an elliptic curve is free and transitive. Therefore

$$
\operatorname{Stab}(\mathcal{D}) \cong \widetilde{\mathrm{GL}^{+}(2 ; \mathbb{R})} \cong \mathbb{C} \times \mathbb{H} \text {. }
$$

By Lemma 2.7 and Lemma 2.12, the systolic ratio

$$
\frac{\operatorname{sys}(\sigma)^{2}}{\operatorname{vol}(\sigma)}
$$

is invariant under the free $\mathbb{C}$-action on the space of stability conditions. Hence we only need to compute the ratio on the quotient $\operatorname{space} \operatorname{Stab}(\mathcal{D}) / \mathbb{C} \cong \mathbb{H}$. The quotient space $\operatorname{Stab}(\mathcal{D}) / \mathbb{C} \cong \mathbb{H}$ can be parametrized by the normalized stability conditions as follows. Let $\tau=\beta+i \omega \in \mathbb{H}$ where $\beta \in \mathbb{R}$ and $\omega>0$. The associated normalized stability condition $\sigma_{\tau}$ is given by:

- Central charge: $\mathcal{Z}_{\tau}(F)=-\operatorname{deg}(F)+\tau \cdot \operatorname{rk}(F)$.

- For $0<\phi \leq 1$, the (semi)stable objects $\mathcal{P}_{\tau}(\phi)$ are the slope-(semi)stable coherent sheaves whose central charges lie in the ray $\mathbb{R}_{>0} \cdot e^{i \pi \phi}$.

- For other $\phi \in \mathbb{R}$, define $\mathcal{P}_{\tau}(\phi)$ by the property $\mathcal{P}_{\tau}(\phi+1)=\mathcal{P}_{\tau}(\phi)[1]$.

Note that there is no wall-crossing phenomenon in the elliptic curve case, i.e., the set of all Bridgeland (semi)stable objects is the same for any stability condition. This makes the computation of categorical systole easier.

To compute the systole of $\sigma_{\tau}$, by Lemma 2.7 (1), we need to know the central charges of all the stable objects of $\sigma_{\tau}$, which are the slope-stable coherent sheaves. Recall that if $F$ is a slope-stable coherent sheaf on $E$, then it is either a vector bundle or a torsion sheaf. The slope-stable vector bundles on an elliptic curve $E$ are well-understood, see for instance $[2,26]$. In particular, we have the following facts:

- Let $F$ be an indecomposable vector bundle of rank $r$ and degree $d$ on an elliptic curve $E$. Then $F$ is slope-stable if and only if $d$ and $r$ are relatively prime.

- Fix a point $x \in E$. For every rational number $\mu=\frac{d}{r}$, where $r>0$ and $(d, r)=1$, there exists a unique slope-stable vector bundle $V_{\mu}$ of rank $r$ and $\operatorname{det}\left(V_{\mu}\right) \cong \mathcal{O}_{E}(d x)$.

Hence the categorical systole is

$$
\begin{aligned}
\operatorname{sys}\left(\sigma_{\tau}\right) & =\min \{1,|-d+\tau r|:(d, r)=1 \text { and } r>0\} \\
& =\left\{|-d+\tau r|:(d, r) \in \mathbb{Z}^{2} \backslash\{(0,0)\}\right\} \\
& =\lambda_{1}\left(L_{\tau}\right),
\end{aligned}
$$

where $\lambda_{1}\left(L_{\tau}\right)$ denotes the least length of a nonzero element in the lattice $L_{\tau}=\langle 1, \tau\rangle$. 
On the other hand, the categorical volume of $\sigma_{\tau}$ has been computed in Example 2.13, which is equals to $2 \omega$. Thus

$$
\sup _{\tau \in \mathbb{H}} \frac{\operatorname{sys}\left(\sigma_{\tau}\right)^{2}}{\operatorname{vol}\left(\sigma_{\tau}\right)}=\frac{1}{2} \cdot \sup _{\tau \in \mathbb{H}} \frac{\lambda_{1}\left(L_{\lambda}\right)^{2}}{\omega} .
$$

Note that $\omega$ is the area of the parallelogram spanned by 1 and $\tau$. Hence the quantity $\sup _{\tau \in \mathbb{H}} \lambda_{1}\left(L_{\lambda}\right)^{2} / \omega$ is the so-called Hermite constant $\gamma_{2}$ of lattices in $\mathbb{R}^{2}$. It is classically known that the Hermite constant is given by $\gamma_{2}=\frac{2}{\sqrt{3}}$ (see for instance [8]). This concludes the proof.

\section{Systolic inequalities for K3 surfaces}

This section is devoted to prove the following main results of the present article.

Theorem 4.1 Let X be a complex projective K3 surface. Then

$$
\operatorname{sys}(\sigma)^{2} \leq C \cdot \operatorname{vol}(\sigma)
$$

for any $\sigma \in \operatorname{Stab}^{\dagger}\left(\mathcal{D}^{b} \operatorname{Coh}(X)\right)$, where

$$
C=\frac{((\rho+2) !)^{2}|\operatorname{disc} \mathrm{NS}(X)|}{2^{\rho}}+4 .
$$

Here $\rho$ and disc denote the rank and the discriminant of the Néron-Severi group $\operatorname{NS}(X)$, respectively.

Theorem 4.2 Let $X$ be a K3 surface of Picard rank one, with $\mathrm{NS}(X)=\mathbb{Z} H$ and $H^{2}=2 n$. Then

$$
\operatorname{sys}(\sigma)^{2} \leq 4(n+1) \cdot \operatorname{vol}(\sigma)
$$

holds for any $\sigma \in \operatorname{Stab}^{\dagger}\left(\mathcal{D}^{b} \operatorname{Coh}(X)\right)$.

\subsection{Reduction to lattice-theoretic problems}

We start with recalling some standard notations. Let $X$ be a smooth complex projective K3 surface and $\mathcal{D}=\mathcal{D}^{b} \operatorname{Coh}(X)$ be its derived category. Sending an object $E \in \mathcal{D}$ to its Mukai vector $v(E)=\operatorname{ch}(E) \sqrt{\operatorname{td}(X)}$ identifies the numerical Grothendieck group of $\mathcal{D}$ with the lattice

$$
\mathcal{N}(\mathcal{D}) \cong H^{0}(X, \mathbb{Z}) \oplus \mathrm{NS}(X) \oplus H^{4}(X, \mathbb{Z}) .
$$

The Mukai pairing on $\mathcal{N}(\mathcal{D})$ is given by

$$
\left(\left(r_{1}, D_{1}, s_{1}\right),\left(r_{2}, D_{2}, s_{2}\right)\right)=D_{1} \cdot D_{2}-r_{1} s_{2}-r_{2} s_{1} .
$$

Since the Mukai pairing on $\mathcal{N}(\mathcal{D})$ is non-degenerate, any group homomorphism $\mathcal{Z}$ : $\mathcal{N}(D) \rightarrow \mathbb{C}$ can be written as $\mathcal{Z}(v)=(\Omega, v)$ for a unique $\Omega \in \mathcal{N}(\mathcal{D}) \otimes \mathbb{C}$. This defines a map

$$
\pi: \operatorname{Stab}(\mathcal{D}) \rightarrow \mathcal{N}(\mathcal{D}) \otimes \mathbb{C}
$$


which sends a stability condition $\sigma=\left(\mathcal{Z}_{\sigma}, \mathcal{P}_{\sigma}\right)$ to the unique element in $\mathcal{N}(\mathcal{D}) \otimes \mathbb{C}$ associated to its central charge $\mathcal{Z}_{\sigma}: \mathcal{N}(\mathcal{D}) \rightarrow \mathbb{C}$.

Define

$$
\begin{aligned}
& \Delta^{+}(\mathcal{D}):=\left\{\delta=(r, D, s) \in \mathcal{N}(\mathcal{D}): \delta^{2}=-2 \text { and } r>0\right\}, \\
& \mathcal{K}(\mathcal{D}):=\left\{\Omega=\exp (\beta+i \omega) \in \mathcal{N}(\mathcal{D}) \otimes \mathbb{C}: \beta, \omega \in \operatorname{NS}(X) \otimes \mathbb{R}, \omega^{2}>0\right\}, \\
& \mathcal{L}(\mathcal{D}):=\left\{\Omega \in \mathcal{K}(\mathcal{D}):(\Omega, \delta) \notin \mathbb{R}_{\leq 0} \text { for all } \delta \in \Delta^{+}(\mathcal{D})\right\} .
\end{aligned}
$$

Now we recall the description of the distinguished connected component $\operatorname{Stab}^{\dagger}(\mathcal{D}) \subset$ $\operatorname{Stab}(\mathcal{D})$ by Bridgeland.

Theorem 4.3 [5] Let $X$ be a complex projective $K 3$ surface and $\mathcal{D}=\mathcal{D}^{b} \operatorname{Coh}(X)$.

(a) Let $V(\mathcal{D}) \subset \operatorname{Stab}(\mathcal{D})$ be the subset consisting of geometric stability conditions constructed via tilting of $\operatorname{Coh}(X)$ in [5, Section 6]. Then the map $\pi$ restricts to give a homeomorphism

$$
\left.\pi\right|_{V(\mathcal{D})}: V(\mathcal{D}) \rightarrow \mathcal{L}(\mathcal{D}) .
$$

(b) Let $U(\mathcal{D}) \subset \operatorname{Stab}(\mathcal{D})$ be the open subset consisting of all geometric stability conditions. For any $\sigma \in U(\mathcal{D})$, there exists a unique $g \in \mathrm{GL}^{+}(2, \mathbb{R})$ such that $\sigma \cdot g \in V(\mathcal{D})$.

(c) Let $\operatorname{Stab}^{\dagger}(\mathcal{D}) \subset \operatorname{Stab}(\mathcal{D})$ be the connected component containing $U(\mathcal{D})$. Every stability condition in $\operatorname{Stab}^{\dagger}(\mathcal{D})$ is mapped into the closure of $U(\mathcal{D})$ by some autoequivalence of $\mathcal{D}$.

The following proposition allows us to compute the categorical systole of a stability condition on $\mathcal{D}$ using only its central charge.

Proposition 4.4 Let $\sigma=\left(\mathcal{Z}_{\sigma}, \mathcal{P}_{\sigma}\right) \in \operatorname{Stab}^{\dagger}(\mathcal{D})$. Then

$$
\operatorname{sys}(\sigma)=\min \left\{\left|\mathcal{Z}_{\sigma}(v)\right|: 0 \neq v \in \mathcal{N}(\mathcal{D}), v^{2}=(v, v) \geq-2\right\} .
$$

Proof Let $v=m v_{0} \in \mathcal{N}(\mathcal{D})$ be a Mukai vector, where $m \in \mathbb{Z}_{>0}$ and $v_{0}$ is primitive. A result of Bayer and Macrì [3, Theorem 6.8], which is based on a previous result of Toda [31], says that if $v_{0}^{2} \geq-2$, then there exists a $\sigma$-semistable object with Mukai vector $v$ for any $\sigma \in \operatorname{Stab}^{\dagger}(\mathcal{D})$. Hence

$$
\begin{aligned}
\operatorname{sys}(\sigma) & =\min \left\{\left|\mathcal{Z}_{\sigma}(E)\right|: E \text { is a } \sigma \text {-semistable object in } \mathcal{D}\right\} \\
& \leq \min \left\{\left|\mathcal{Z}_{\sigma}(v)\right|: 0 \neq v \in \mathcal{N}(\mathcal{D}), v^{2} \geq-2\right\} .
\end{aligned}
$$

On the other hand, for any stable object $E$,

$$
\begin{aligned}
v(E)^{2} & =-\chi(E, E)=-\operatorname{hom}^{0}(E, E)+\operatorname{hom}^{1}(E, E)-\operatorname{hom}^{2}(E, E) \\
& =-2+\operatorname{hom}^{1}(E, E) \geq-2 .
\end{aligned}
$$

Hence

$$
\begin{aligned}
\operatorname{sys}(\sigma) & =\min \left\{\left|\mathcal{Z}_{\sigma}(E)\right|: E \text { is a } \sigma \text {-stable object in } \mathcal{D}\right\} \\
& \geq \min \left\{\left|\mathcal{Z}_{\sigma}(v)\right|: 0 \neq v \in \mathcal{N}(\mathcal{D}), v^{2} \geq-2\right\}
\end{aligned}
$$

This concludes the proof. 
We can now reduce the categorical systolic inequality to a lattice-theoretic problem. To prove Theorem 4.1 and 4.2, one needs to find an upper bound of the systolic ratio

$$
\frac{\operatorname{sys}(\sigma)^{2}}{\operatorname{vol}(\sigma)}
$$

for all $\sigma \in \operatorname{Stab}^{\dagger}(\mathcal{D})$. Since the systolic ratio is a continuous function and is invariant under the actions of autoequivalences, by Theorem 4.3 (3), it suffices to find an upper bound of the systolic ratio on $U(\mathcal{D})$.

By Theorem 4.3 (2), any element in $U(\mathcal{D})$ can be written as $\sigma \cdot g$ for some $\sigma \in V(\mathcal{D})$ and $g \in \mathrm{GL}^{+}(2, \mathbb{R})$. By Lemma 2.7 (4) and the computations in Example 2.15, we have

$$
\frac{\operatorname{sys}(\sigma \cdot g)^{2}}{\operatorname{vol}(\sigma \cdot g)} \leq \frac{\left(1+\left|t_{2}\right|\right)^{2}}{1+\left|t_{2}\right|^{2}} \cdot \frac{\operatorname{sys}(\sigma)^{2}}{\operatorname{vol}(\sigma)}<4 \cdot \frac{\operatorname{sys}(\sigma)^{2}}{\operatorname{vol}(\sigma)}
$$

since $\left|t_{2}\right|<1$ (recall the notations in Lemma 2.7). Therefore, it is enough to find an upper bound of the systolic ratio on $V(\mathcal{D})$.

By Theorem 4.3 (1), the central charges of stability conditions in $V(\mathcal{D})$ are of the form

$$
\mathcal{Z}_{\sigma}(v)=(\exp (\beta+i \omega), v)
$$

where $\beta, \omega \in \mathrm{NS}(X) \otimes \mathbb{R}$ and $\omega^{2}>0$. By Example 2.15, the volume of the stability conditions of this form is $2 \omega^{2}$. On the other hand, the categorical systole can be computed by Proposition 4.4 given the central charge:

$$
\begin{aligned}
\operatorname{sys}(\sigma) & =\min \left\{\left|\mathcal{Z}_{\sigma}(v)\right|: 0 \neq v \in \mathcal{N}(\mathcal{D}), v^{2}=(v, v) \geq-2\right\} \\
& =\min _{\substack{(r, D, s) \neq(0,0,0) \\
D^{2}-2 r s \geq-2}}\left\{\left|\left(s+\beta . D+\frac{1}{2}\left(\beta^{2}-\omega^{2}\right) r\right)+i(\omega .(D+r \beta))\right|\right\}
\end{aligned}
$$

Hence, Theorem 4.1 and 4.2 can be obtained by proving the following lattice-theoretic statements.

Proposition 4.5 For any $\beta, \omega \in \mathrm{NS}(X) \otimes \mathbb{R}$ with $\omega^{2}>0$ and any $C>\frac{(\rho+2) ! \sqrt{|\operatorname{discNS}(X)|}}{2^{(\rho+1) / 2}}$, there exists $(r, D, s) \in \mathcal{N}(\mathcal{D}) \backslash\{(0,0,0)\}$ satisfying

(a) $|\omega \cdot(D+r \beta)| \leq C \sqrt{\omega^{2}}$;

(b) $\left|s+\beta \cdot D+\frac{1}{2}\left(\beta^{2}-\omega^{2}\right) r\right| \leq \sqrt{2 \omega^{2}}$;

(c) $D^{2}-2 r s \geq-2$.

Proposition 4.6 For any $\beta \in \mathbb{R}$ and $\omega>0$, there exists $(r, d, s) \in \mathbb{Z}^{3} \backslash\{(0,0,0)\}$ not all zero satisfying

(a)

$$
\frac{\left|s+2 n(\beta+i \omega) d+n(\beta+i \omega)^{2} r\right|^{2}}{4 n \omega^{2}}<n+1 ;
$$

(b) $n d^{2}-r s \geq-1$.

We will prove these two propositions in the next two subsections. 


\subsection{Systolic inequality for K3 surfaces of Picard rank one}

We prove Proposition 4.6 in this subsection. Note that the method in this proof does not work for K3 surfaces with higher Picard rank, due to the indefiniteness of the intersection pairing on $\mathrm{NS}(X)$ for $\rho(X)>1$.

In order to find such triple $(r, d, s)$ in Proposition 4.6 for small $\omega$, we need the following technical lemma.

Lemma 4.7 For any real number $\beta$ and any $0<\omega<\frac{1}{\sqrt{n}}$, there exists integers $(r, d, s)$ such that:

$$
\begin{aligned}
& 1 \leq r \leq \frac{1}{\sqrt{n} \omega}, \\
& \left|s+2 n \beta d+n \beta^{2} r\right|<\sqrt{n} \omega, \\
& 0 \leq n d^{2}-s r \leq n
\end{aligned}
$$

Proof Let $l=\left\lfloor\frac{1}{\sqrt{n} \omega}\right\rfloor+1$. For each $1 \leq j \leq l$, choose $d_{j} \in \mathbb{Z}$ such that

$$
-\frac{1}{2}<d_{j}+j \beta \leq \frac{1}{2}
$$

Consider the real numbers $\left\{2 n \beta d_{j}+n \beta^{2} j\right\}_{1 \leq j \leq l}$ modulo 1 . There is at least a pair $\left(2 n \beta d_{j}+n \beta^{2} j, 2 n \beta d_{k}+n \beta^{2} k\right)$ has distance less than or equals to $1 / l$ modulo 1 . Say $j>k$ without loss of generality. We choose $r=j-k, d=d_{j}-d_{k}$, and choose $s$ to be the integer closest to $-2 n \beta d-n \beta^{2} r$. Then

$$
1 \leq r \leq\left\lfloor\frac{1}{\sqrt{n} \omega}\right\rfloor
$$

and

$$
\left|s+2 n \beta d+n \beta^{2} r\right| \leq \frac{1}{\left\lfloor\frac{1}{\sqrt{n} \omega}\right\rfloor+1}<\sqrt{n} \omega .
$$

Let $\epsilon=s+2 n \beta d+n \beta^{2} r$. Then

$$
\begin{aligned}
n d^{2}-s r & =n d^{2}-\left(-2 n \beta d-n \beta^{2} r+\epsilon\right) r \\
& =n(d+r \beta)^{2}-r \epsilon .
\end{aligned}
$$

We have

$$
(d+r \beta)^{2}=\left(\left(d_{j}+j \beta\right)-\left(d_{k}+k \beta\right)\right)^{2}<1
$$

and

$$
|r \epsilon|<\frac{1}{\sqrt{n} \omega} \cdot \sqrt{n} \omega=1 .
$$

Hence $-1<n d^{2}-s r<n+1$. Since it is an integer, thus $0 \leq n d^{2}-s r \leq n$.

We can now prove Proposition 4.6. 
Proof of Proposition 4.6 If $\omega \geq \frac{1}{\sqrt{n}}$, one can simply take the class of skyscraper sheaves $(r, d, s)=(0,0,1)$ and check that it satisfies the required conditions. If $\omega<\frac{1}{\sqrt{n}}$, we choose $(r, d, s)$ as in Lemma 4.7. Then it satisfies $s r<n d^{2}+1$ and

$$
\begin{aligned}
\frac{\left|s+2 n(\beta+i \omega) d+n(\beta+i \omega)^{2} r\right|^{2}}{4 n \omega^{2}} & =\frac{1}{4 n}\left(\frac{s+2 n \beta d+n \beta^{2} r}{\omega}+n \omega r\right)^{2}+\left(n d^{2}-r s\right) \\
& <\frac{1}{4 n}(\sqrt{n}+\sqrt{n})^{2}+n=n+1 .
\end{aligned}
$$

Remark 4.8 (Spherical systole) The notion of spherical objects in a triangulated category was introduced by Seidel and Thomas [30]. These objects are the categorical analogue of Lagrangian spheres in derived Fukaya categories. One can define the spherical systole of a Bridgeland stability condition $\sigma$ on a triangulated category $\mathcal{D}$ as the minimum among the masses of spherical objects:

$$
\operatorname{sys}_{\text {sph }}(\sigma):=\min \left\{m_{\sigma}(S): S \text { is a spherical object in } \mathcal{D}\right\} .
$$

It is not hard to show that the notions of spherical systole and categorical systole coincide for stability conditions on elliptic curves. However, this is not true for the derived categories of K3 surfaces. The following proposition shows that the systolic inequality does not hold for spherical systole on K3 surfaces.

Proposition 4.9 Let $X$ be a K3 surface of Picard rank one and $\mathcal{D}=\mathcal{D}^{b} \operatorname{Coh}(X)$ be its derived category of coherent sheaves. Then

$$
\sup _{\sigma \in \operatorname{Stab}^{\dagger}(\mathcal{D})} \frac{\operatorname{sys}_{\mathrm{sph}}(\sigma)^{2}}{\operatorname{vol}(\sigma)}=+\infty .
$$

Proof Let $\mathrm{NS}(X)=\mathbb{Z} H$ and $H^{2}=2 n$. Let $\omega H \in \mathrm{NS}(X) \otimes \mathbb{R}$ be an ample class. Then $\mathcal{Z}=(\exp (i \omega H),-)$ gives the central charge of a stability condition in $\operatorname{Stab}^{\dagger}(\mathcal{D})$ if $\omega>1$ ([5, Lemma 6.2]). By Proposition 4.4 and Example 2.15,

$$
\sup _{\sigma \in \operatorname{Stab}^{\dagger}(\mathcal{D})} \frac{\operatorname{sys}_{\operatorname{sph}}(\sigma)^{2}}{\operatorname{vol}(\sigma)} \geq \sup _{\omega>1} \frac{\min \left\{|(\exp (i \omega H), v)|^{2}: v^{2}=-2\right\}}{4 n \omega^{2}} .
$$

Here we use the fact that the Mukai vector of a spherical object $S$ satisfies $v(S)^{2}=$ $-\operatorname{hom}^{0}(S, S)+\operatorname{hom}^{1}(S, S)-\operatorname{hom}^{2}(S, S)=-2$.

Let $v=(r, d H, s) \in \mathbb{Z} \oplus \mathrm{NS}(X) \oplus \mathbb{Z}$ be a vector satisfying $v^{2}=2 n d^{2}-2 r s=-2$. Then $r s \neq 0$ and $r, s$ are both positive or both negative. Hence

$$
\begin{aligned}
\sup _{\sigma \in \operatorname{Stab}^{\dagger}(\mathcal{D})} \frac{\operatorname{sys}_{\mathrm{sph}}(\sigma)^{2}}{\operatorname{vol}(\sigma)} & \geq \sup _{\omega>1} \frac{\min \left\{|(\exp (i \omega H), v)|^{2}: v^{2}=-2\right\}}{4 n \omega^{2}} \\
& =\sup _{\omega>1} \min _{n d^{2}-r s=-1} \frac{1}{4 n}\left(\frac{s}{\omega}+n \omega r\right)^{2}-1 \\
& \geq \sup _{\omega>1} \frac{1}{4 n}\left(\frac{1}{\omega}+n \omega\right)^{2}-1=+\infty .
\end{aligned}
$$


Remark 4.10 In the proof of Proposition 4.6, we do not make use of the Mukai vectors of spherical objects. Recall that

$$
\operatorname{sys}(\sigma)=\min \left\{\left|\mathcal{Z}_{\sigma}(v)\right|: v^{2} \geq-2, v \neq 0\right\},
$$

but we only use the Mukai vectors that satisfy $0 \leq v^{2} \leq 2 n$ to prove the systolic inequality (c.f. Lemma 4.7).

\subsection{Systolic inequality for general K3 surfaces}

We prove Proposition 4.5 in this subsection. The proof is based on a classical result on the existence of integer points by Minkowski.

Theorem 4.11 (Minkowski) Every convex set in $\mathbb{R}^{n}$ which is symmetric with respect to the origin and has volume greater than $2^{n}$ contains a non-zero integer point.

Proof of Proposition 4.5 We fix an identification $\mathrm{NS}(X) \cong \mathbb{Z}^{\rho}$. For any $\omega \in \mathrm{NS}(X) \otimes \mathbb{R}$ with $\omega^{2}>0$, the intersection pairing restricts on $\langle\omega\rangle^{\perp} \subset \mathbb{R}^{\rho}$ is negative definite by Hodge index theorem. Choose

$$
D_{1}, \ldots, D_{\rho-1} \in\langle\omega\rangle^{\perp} \subset \mathbb{R}^{\rho}
$$

such that $D_{i} \cdot D_{j}=0$ for all $i \neq j$, and $D_{i}^{2}=-1$ for all $i$.

Consider the following vectors in $\mathbb{R}^{\rho+2} \cong \mathbb{R} \oplus(\mathrm{NS}(X) \otimes \mathbb{R}) \oplus \mathbb{R}$ :

$$
\begin{aligned}
v_{1} & =\sqrt{2} \cdot\left(0, D_{1},-\beta \cdot D_{1}\right), \\
v_{2} & =\sqrt{2} \cdot\left(0, D_{2},-\beta \cdot D_{2}\right), \\
\ldots & =\sqrt{2} \cdot\left(0, D_{\rho-1},-\beta \cdot D_{\rho-1}\right), \\
v_{\rho-1} & =\sqrt{2} \cdot\left(\frac{1}{\sqrt{\omega^{2}}}, \frac{-\beta}{\sqrt{\omega^{2}}}, \frac{\beta^{2}+\omega^{2}}{2 \sqrt{\omega^{2}}}\right), \\
v_{\rho} & \left.=\sqrt{\omega^{2}}, \frac{-\beta \cdot \omega}{\sqrt{\omega^{2}}}\right), \\
v_{\rho+1} & =C \cdot\left(0, \frac{\omega}{\sqrt{\omega^{2}}}\right) \\
v_{\rho+2} & =\sqrt{2} \cdot\left(0,0, \sqrt{\omega^{2}}\right),
\end{aligned}
$$

where $C$ is any number larger than $\frac{(\rho+2) ! \sqrt{|\operatorname{discNS}(X)|}}{2(\rho+1) / 2}$.

Consider the following convex set in $\mathbb{R}^{\rho+2}$ which is symmetric with respect to the origin:

$$
\mathcal{C}:=\left\{\sum_{i=1}^{\rho+2} e_{i} v_{i}: \sum_{i=1}^{\rho+2}\left|e_{i}\right| \leq 1\right\} .
$$

One can check that the volume of $\mathcal{C}$ is greater than $2^{\rho+2}$. Therefore to prove Proposition 4.5, it suffices to show that any vector in $\mathcal{C}$ satisfies the following conditions:

(a) $|\omega \cdot(D+r \beta)| \leq C \sqrt{\omega^{2}}$;

(b) $\left|s+\beta \cdot D+\frac{1}{2}\left(\beta^{2}-\omega^{2}\right) r\right| \leq \sqrt{2 \omega^{2}}$;

(c) $D^{2}-2 r s \geq-2$. 
Observe that the vectors $v_{1}, v_{2}, \ldots, v_{\rho+2}$ satisfy all three conditions. Since the first two conditions are linear in $(r, D, s) \in \mathbb{R}^{\rho+2}$, hence are satisfied by all the vectors in $\mathcal{C}$.

Let $(r, D, s)=\sum_{i=1}^{\rho+2} e_{i} v_{i} \in \mathcal{C}$. Then

$$
\begin{aligned}
D^{2}-2 r s & =e_{\rho+1}^{2} C^{2}-2\left(e_{1}^{2}+\cdots+e_{\rho}^{2}\right)-4 e_{\rho} e_{\rho+2} \\
& \geq-2\left(e_{1}^{2}+\cdots+e_{\rho-1}^{2}\right)-2\left(e_{\rho}+e_{\rho+2}\right)^{2} \\
& \geq-2
\end{aligned}
$$

since $\sum_{i=1}^{\rho+2}\left|e_{i}\right| \leq 1$. This concludes the proof of Proposition 4.5 by Minkowski's theorem.

\section{Future studies}

\section{Applications in symplectic geometry}

Recall the two questions we proposed in the introduction.

Question 5.1 (see Question 1.2) Let $Y$ be a Calabi-Yau manifold and $\omega$ be a symplectic form on $Y$. Does there exist a constant $C=C(Y, \omega)>0$ such that

$$
\operatorname{sys}(Y, \omega, \Omega)^{2} \leq C \cdot\left|\int_{Y} \Omega \wedge \bar{\Omega}\right|
$$

holds for any holomorphic top form $\Omega$ on $Y$ ?

Question 5.2 (see Question 1.6) Let $X$ be a Calabi-Yau manifold and $\Omega$ be a complex structure on $X$. Let $\mathcal{D}=\mathcal{D}^{b} \operatorname{Coh}(X, \Omega)$ be its derived category of coherent sheaves. Does there exist a constant $C=C(X, \Omega)>0$ such that

$$
\operatorname{sys}(\sigma)^{2} \leq C \cdot \operatorname{vol}(\sigma)
$$

holds for any $\sigma \in \operatorname{Stab}^{\dagger}(\mathcal{D})$ ?

In the present article, we give an affirmative answer to Question 5.2 for any complex projective K3 surface $X$, and find an explicit systolic constant $C$ which depends only on the rank and discriminant of $\mathrm{NS}(X)$. Now we discuss how this result can be used to answer Question 5.1, via the homological mirror symmetry conjecture proposed by Kontsevich.

Conjecture 5.3 [18] For any Calabi-Yau manifold with a symplectic structure $(Y, \omega)$, there exists a Calabi-Yau manifold with a complex structure $(X, \Omega)$ such that there is an equivalence between triangulated categories

$$
\mathcal{D}^{b} \operatorname{Fuk}(Y, \omega) \cong \mathcal{D}^{b} \operatorname{Coh}(X, \Omega) .
$$

The conjecture has been proved in several cases, see for instance [27-29]. In particular, for any $\mathrm{K} 3$ surface $Y$ and a symplectic form $\omega$ on $Y$, it is expected that there exists a $\mathrm{K} 3$ surface $X$ with a complex structure $\Omega$ such that the above equivalence of categories holds.

Corollary 5.4 Assume Conjecture 2.3 and 5.3 hold for all K3 surfaces. Also assume that the space of stability conditions on $\mathcal{D}^{b} \mathrm{Coh}(X)$ is connected for any projective K3 surface $X$. Then Question 5.1 has an affirmative answer for any $K 3$ surface $Y$. 
Proof Conjecture 2.3 holds for $\mathcal{D}^{b} \operatorname{Fuk}(Y, \omega)$ implies that for any holomorhpic 2-form $\Omega_{Y}$ on $Y$, there is an associated Bridgeland stability condition $\sigma_{\Omega_{Y}}$ on the derived Fukaya category of $(Y, \omega)$ such that

$$
\operatorname{sys}\left(Y, \omega, \Omega_{Y}\right)=\operatorname{sys}\left(\sigma_{\Omega_{Y}}\right) \text { and }\left|\int_{Y} \Omega_{Y} \wedge \bar{\Omega}_{Y}\right|=\operatorname{vol}\left(\sigma_{\Omega_{Y}}\right) .
$$

Assuming the validity of Conjecture 5.3 for $\mathrm{K} 3$ surface $(Y, \omega)$, then there exists a $\mathrm{K} 3$ surface with a complex structure $(X, \Omega)$ such that

$$
\mathcal{D}^{b} \operatorname{Fuk}(Y, \omega) \cong \mathcal{D}^{b} \operatorname{Coh}(X, \Omega) .
$$

Therefore $\sigma_{\Omega_{Y}}$ induces a Bridgeland stability condition on $\mathcal{D}^{b} \operatorname{Coh}(X, \Omega)$. By Theorem 1.7 and the assumption that $\operatorname{Stab}\left(\mathcal{D}^{b} \operatorname{Coh}(X)\right)$ is connected, we have

$$
\operatorname{sys}\left(\sigma_{\Omega_{Y}}\right)^{2} \leq C \cdot \operatorname{vol}\left(\sigma_{\Omega_{Y}}\right),
$$

where $C=\frac{((\rho+2) !)^{2}|\operatorname{disc} \mathrm{NS}(X)|}{2^{\rho}}+4$. Hence, the inequality

$$
\operatorname{sys}\left(Y, \omega, \Omega_{Y}\right)^{2} \leq C \cdot\left|\int_{Y} \Omega_{Y} \wedge \bar{\Omega}_{Y}\right|
$$

holds for any holomorphic 2-form $\Omega_{Y}$ on $Y$.

\section{Systolic inequality for Calabi-Yau threefolds}

The existence of Bridgeland stability conditions on quintic Calabi-Yau threefolds is proved by Li recently [21]. It would be interesting to investigate whether the categorical systolic inequality

$$
\operatorname{sys}(\sigma)^{2} \leq C \cdot \operatorname{vol}(\sigma)
$$

continues to hold for Calabi-Yau threefolds. Note that the categorical volumes of geometric stability conditions on quintic Calabi-Yau threefolds near the "large volume limit" were computed in [10, Section 4.4]. Hence the main difficulty lies in determining the categorical systoles. It would be nice if properties similar to Proposition 4.4 hold for Calabi-Yau threefolds.

\section{Categorical systole as topological Morse function}

It is proved by Akrout [1] that the systole of Riemann surfaces is a topological Morse function on the Teichmüller space. In fact, Akrout shows that a "generalized systolic function" defined locally as the minimum of a finite number of functions with positive definite Hessians is a topological Morse function, and then apply a result of Wolpert [32] that length functions have positive definite Hessians with respect to the Weil-Petersson metric on the Teichmüller space.

Motivated by the correspondence between flat surfaces and stability conditions, it would be interesting to show that the categorical systole is a topological Morse function on the space of Bridgeland stability conditions, and then deduce some topological properties of the quotient $\operatorname{space} \operatorname{Stab}(\mathcal{D}) / \operatorname{Aut}(\mathcal{D})$. Since there also is a categorical analogue of Weil-Petersson metric on the space of stability conditions studied in [10], one might be able to follow the same approach as Akrout's proof. We hope to come back to this question in the future. 
Acknowledgements The author would like to thank Jayadev Athreya, Simion Filip, Fabian Haiden, Pei-Ken Hung, Atsushi Kanazawa, Kuan-Wen Lai, Heather Lee, Yu-Shen Lin, Emanuele Macrì, Nikolay Moshchevitin, Shing-Tung Yau and Xiaolei Zhao for helpful discussions and correspondences. This work was partially supported by Simons Collaboration Grant on Homological Mirror Symmetry. The author would also like to thank an anonymous referee for helpful comments and suggestions.

Open Access This article is licensed under a Creative Commons Attribution 4.0 International License, which permits use, sharing, adaptation, distribution and reproduction in any medium or format, as long as you give appropriate credit to the original author(s) and the source, provide a link to the Creative Commons licence, and indicate if changes were made. The images or other third party material in this article are included in the article's Creative Commons licence, unless indicated otherwise in a credit line to the material. If material is not included in the article's Creative Commons licence and your intended use is not permitted by statutory regulation or exceeds the permitted use, you will need to obtain permission directly from the copyright holder. To view a copy of this licence, visit http://creativecommons.org/licenses/by/4.0/.

\section{References}

1. Akrout, H.: Singularités topologiques des systoles généralisées. Topology 42(2), 291-308 (2003)

2. Atiyah, M.F.: Vector bundles over an elliptic curve. Proc. Lond. Math. Soc. 3(7), 414-452 (1957)

3. Bayer, A., Macrì, E.: Projectivity and birational geometry of Bridgeland moduli spaces. J. Am. Math. Soc. 27(3), 707-752 (2014)

4. Bridgeland, T.: Stability conditions on triangulated categories. Ann. Math. (2), 166(2), 317-345 (2007)

5. Bridgeland, T.: Stability conditions on $K 3$ surfaces. Duke Math. J. 141(2), 241-291 (2008)

6. Bridgeland, T.: Spaces of stability conditions. In: Algebraic geometry-Seattle 2005. Part 1, volume 80 of Proc. Sympos. Pure Math., pp. 1-21. Amer. Math. Soc., Providence, RI (2009)

7. Bridgeland, T., Smith, I.: Quadratic differentials as stability conditions. Publ. Math. Inst. Hautes Études Sci. 121, 155-278 (2015)

8. Cassels, J.W.S.: An Introduction to the Geometry of Numbers. Classics in Mathematics. Springer, Berlin (1997)

9. Douglas, M.R., Reinbacher, R., Yau, S.-T.: Branes. Bogomolov and beyond, bundles and attractors. arXiv:math/0604597 (2006)

10. Fan, Y.-W., Kanazawa, A., Yau, S.-T.: Weil-Petersson geometry on the space of Bridgeland stability conditions. arXiv:1708.02161 (2017)

11. Ginzburg, V.: Calabi-Yau algebras. arXiv:math/0612139 (2006)

12. Gaiotto, D., Moore, G.W., Neitzke, A.: Wall-crossing, Hitchin systems, and the WKB approximation. Adv. Math. 234, 239-403 (2013)

13. Haiden, F.: An extension of the Siegel space of complex abelian varieties and conjectures on stability structures. arXiv:1808.06364 (2018)

14. Haiden, F., Katzarkov, L., Kontsevich, M.: Flat surfaces and stability structures. Publ. Math. Inst. Hautes Études Sci. 126, 247-318 (2017)

15. Joyce, D.: Conjectures on Bridgeland stability for Fukaya categories of Calabi-Yau manifolds, special Lagrangians, and Lagrangian mean curvature flow. EMS Surv. Math. Sci. 2(1), 1-62 (2015)

16. Katz, M.G.: Systolic geometry and topology, volume 137 of Mathematical Surveys and Monographs. American Mathematical Society, Providence, RI. With an appendix by J. P. Solomon (2007)

17. Keller, B.: Deformed Calabi-Yau completions. J. Reine Angew. Math. 654, 125-180 (2011) [With an appendix by M. Van den Bergh]

18. Kontsevich, M.: Homological algebra of mirror symmetry. In: Proceedings of the International Congress of Mathematicians, vol. 1, 2 (Zürich: pp. 120-139. Birkhäuser, Basel (1994)

19. Kontsevich, M., Soibelman, Y.: Stability structures, motivic Donaldson-Thomas invariants and cluster transformations. arXiv:0811.2435 (2008)

20. Kontsevich, M., Soibelman, Y.: Wall-crossing structures in Donaldson-Thomas invariants, integrable systems and mirror symmetry. In: Homological mirror symmetry and tropical geometry, volume 15 of Lect. Notes Unione Mat. Ital., pp. 197-308. Springer, Cham (2014)

21. Li, C.: On stability conditions for the quintic threefold. Invent. Math. 218(1), 301-340 (2019)

22. Macrì, E.: Stability conditions on curves. Math. Res. Lett. 14(4), 657-672 (2007)

23. Moore, G.W.: Arithmetic and attractors. arXiv:hep-th/9807087 (1998)

24. Okada, S.: Stability manifold of $\mathbb{P}^{1}$. J. Algebr. Geom. 15(3), 487-505 (2006) 
25. Pacini, T.: Extremal length in higher dimensions and complex systolic inequalities. arXiv:1904.07807 (2019)

26. Polishchuk, A.: Abelian Varieties, Theta Functions and the Fourier Transform. Cambridge Tracts in Mathematics, vol. 153. Cambridge University Press, Cambridge (2003)

27. Polishchuk, A., Zaslow, E.: Categorical mirror symmetry: the elliptic curve. Adv. Theor. Math. Phys. 2(2), 443-470 (1998)

28. Seidel, P.: Homological mirror symmetry for the quartic surface. Mem. Am. Math. Soc. 236(1116), vi+129 (2015)

29. Sheridan, N.: Homological mirror symmetry for Calabi-Yau hypersurfaces in projective space. Invent. Math. 199(1), 1-186 (2015)

30. Seidel, P., Thomas, R.: Braid group actions on derived categories of coherent sheaves. Duke Math. J. 108(1), 37-108 (2001)

31. Toda, Y.: Moduli stacks and invariants of semistable objects on $K 3$ surfaces. Adv. Math. 217(6), 27362781 (2008)

32. Wolpert, S.A.: Geodesic length functions and the Nielsen problem. J. Differ. Geom. 25(2), 275-296 (1987)

Publisher's Note Springer Nature remains neutral with regard to jurisdictional claims in published maps and institutional affiliations. 